\title{
Concurrent Presentation of Neurobrucellosis and Spinal Cyst Hydatid: A Case Report
}

\section{Ceren Yalnız, Kaan Meric, Hasan Gundogdu, Sibel Aydın, Zeynep Gamze Kılıcoglu and Mehmet Masum Simsek}

Haydarpasa Numune Training and Research Hospital of Radiology, Istanbul, Turkey

Corresponding author: Ceren Yalnız, Haydarpasa Numune Training and Research Hospital of Radiology, Tıbbiye Cad. No: 2334668 Uskudar / Istanbul, Turkey, Tel: 00905057084210; Fax:00902163360565; E-mail: cerenyalniz@gmail.com

Received: May 19, 2016; Accepted: Jun 27, 2016; Published: Jun 30, 2016

\section{Abstract}

Introduction: Brucellosis and cyst hydatid are highly endemic to Turkey. Although these diseases are mostly seen separately, it is also possible to see them together in the same patient. In this case report, we present a patient diagnosed with neurobrucellosis and vertebral cyst hydatid at the same time.

Case report: A thirty-five-year-old woman presented with progressive weakness, and numbness in both the legs, headache and fever. The patient came from a province where brucellosis was endemic and her Brucella agglutination tests were positive. The abscess formation encountered in her spinal MRI and milimetric hyperintense lesions on T2-weighted images in white matter in her cranial MRI are interpreted as different presentations of brucellosis. After antibiotic therapy for brucellosis, cranial symptoms dramatically improved. Since the vertebral lesion persisted despite of the medical therapy, it was operated and the pathology of the lesion was reported as hydatid cyst.

Discussion: Spinal cyst hydatid is the most common bone involvement of cyst hydatid and it is usually difficult to distinguish from other infectious diseases such as tuberculosis, brucellosis and chronic osteomyelitis. Although very rare, spinal cyst hydatid might be accompanied by a secondary infection and present like an abscess, making the differential diagnosis almost impossible. In such cases histopathological evaluation is extremely important.

Brucellosis is another disease commonly encountered in Turkey and the other Mediterranean countries. Inflammation, vascular insult and white matter changes are the imaging abnormalities encountered in neurobrucellosis. After appropriate therapy, complete clinical and radiologic improvement is possible. Brucellosis and cyst hydatid might be encountered in the same patient, especially in endemic areas. In our case, neurobrucellosis and spinal hydatid cyst presented at the same time, making the differential diagnosis highly difficult.
Conclusion: Hydatid cyst and brucellosis may present at the same time but in different ways. In diagnosis process, if we try to explain all symptoms and lesions with the diagnosis of hydatid cyst or brucellosis solely, we may end up missing one of them.

Keywords: Neurobrucellosis; Cyst hydatid; Magnetic Resonance Imaging (MRI)

\section{Introduction}

Brucellosis and cyst hydatid are serious infectious diseases, highly endemic to Turkey. Although these diseases are mostly seen separately, it is also possible to see them together in the same patient [1]. In this case report, we present a patient diagnosed with neurobrusellosis and vertebral cyst hydatid at the same time.

\section{Case Report}

A thirty-five-year-old woman from Kars province of eastern Turkey, with no previously known illnesses, presented with progressive weakness, and numbness in both the legs, headache and fever.
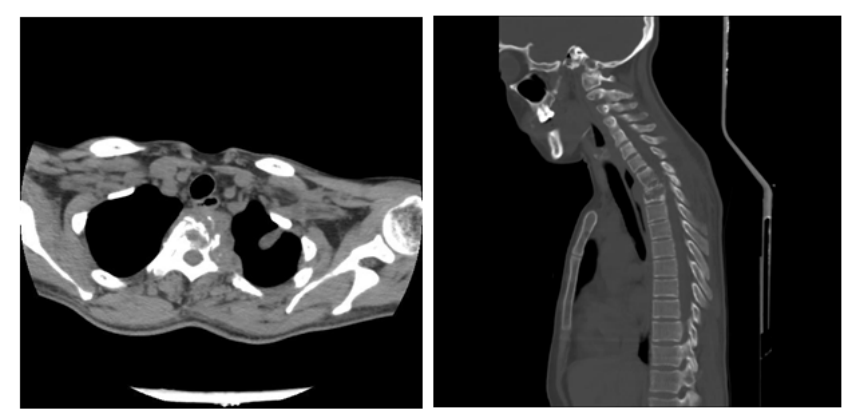

Figure 1 Thoracic computed tomography (CT) scans revealed a hypo-dense mass lesion which caused destruction of first, second and third thoracic vertebrae and extended to the intervertebral disc space and paravertebral tissue. 
Thoracic computed tomography (CT) scans revealed a hypodense mass lesion which caused destruction of first, second and third thoracic vertebrae and extended to the intervertebral disc space and paravertebral tissue (Figure 1).

Magnetic resonance imaging (MRI) of the thoracic region revealed the lesion was hypo-intense on T1-weighted (Figure 2) images and heterogeneously hyper-intense on T2-weighted images (Figure 3). The lesion showed peripheral enhancement, following intravenous gadolinium (Figure 4). The lesion destroyed the corpuses, laminae and pedicules of the first and second thoracic vertebrae and the third vertebral corpus partially. Our primary diagnoses were infectious processes/ abscess.

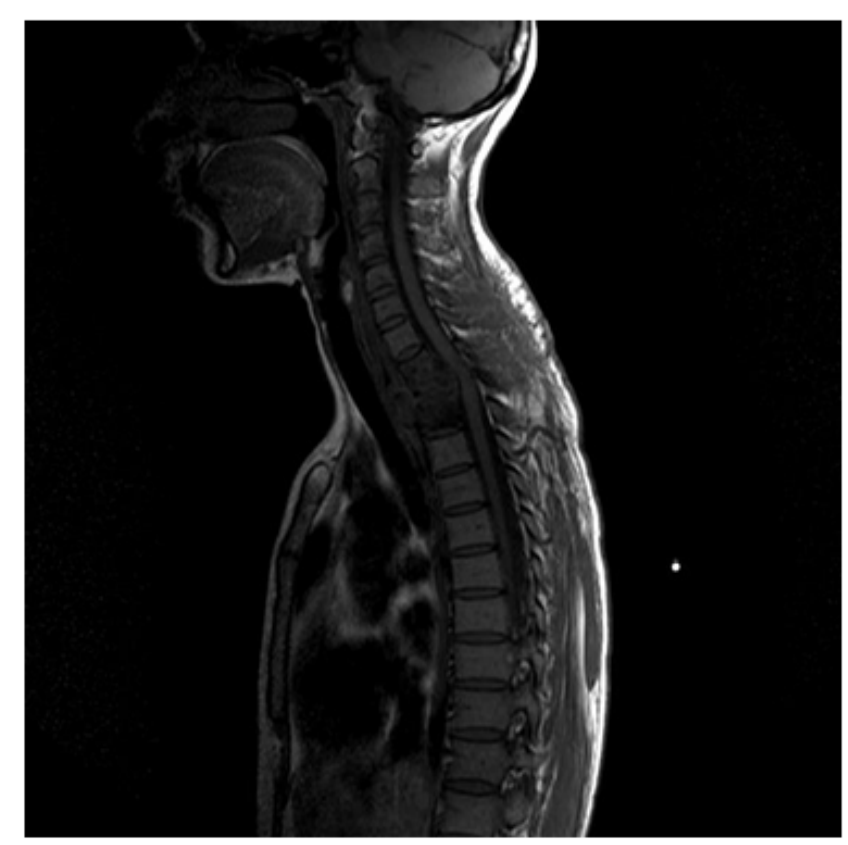

Figure 2 Magnetic resonance imaging (MRI) of the thoracic region revealed the lesion was hypo-intense on T1-weighted image.
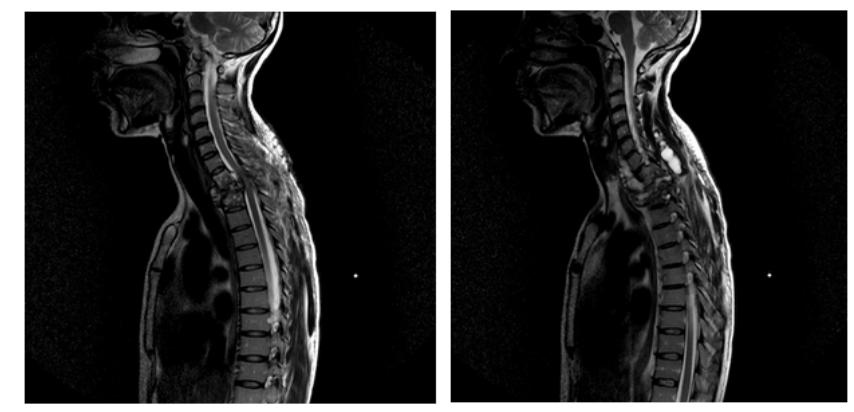

Figure 3 Magnetic resonance imaging (MRI) of the thoracic region revealed heterogeneously hyper-intense on T2weighted images.

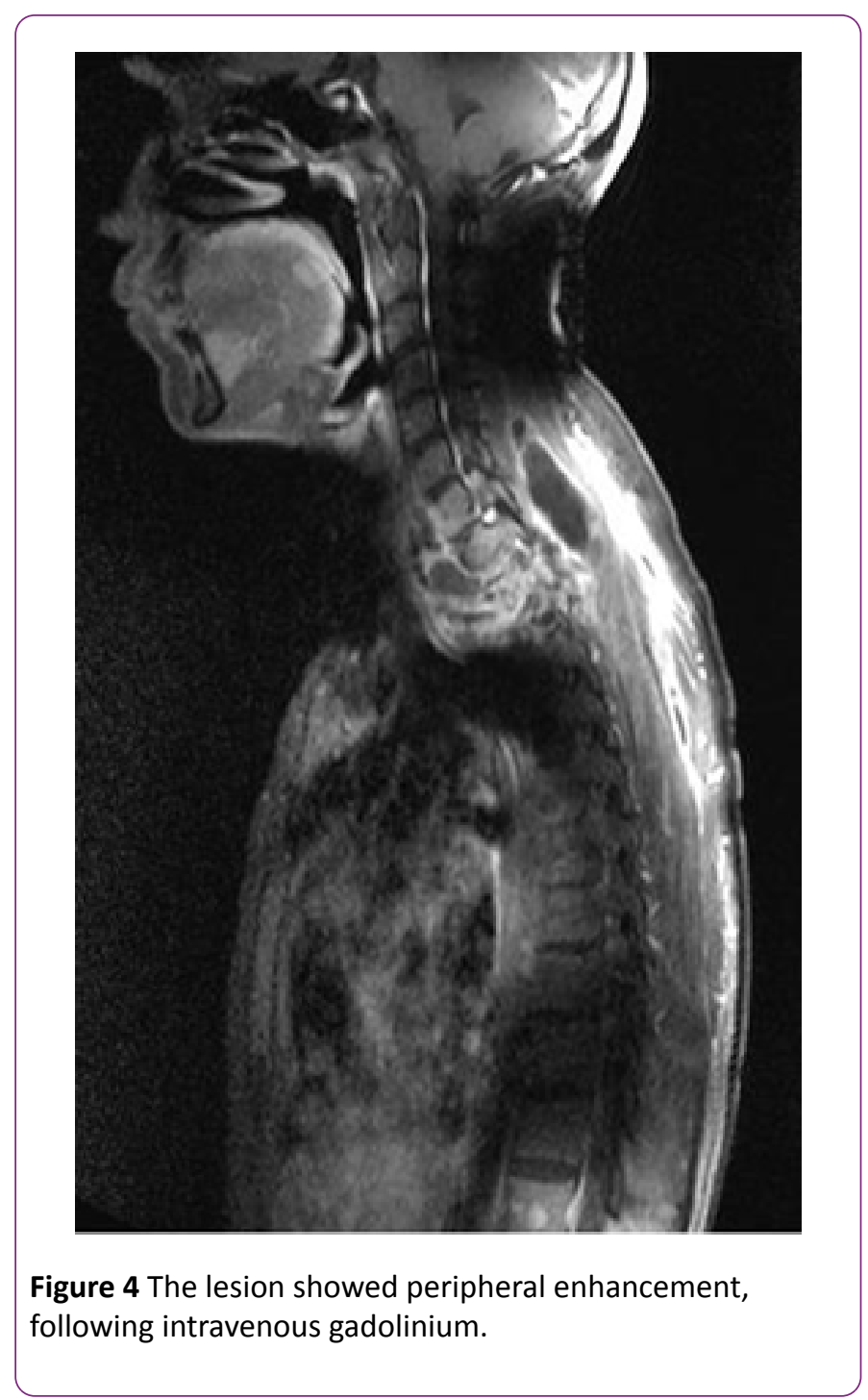

The patient also went through cranial MRI for her neurologic symptoms like headache and memory problems. Cranial MRI revealed milimetric hyper-intense lesions on T2-weighted and FLAIR images in the centrum semiovale and periventricular white matter (Figure 5).
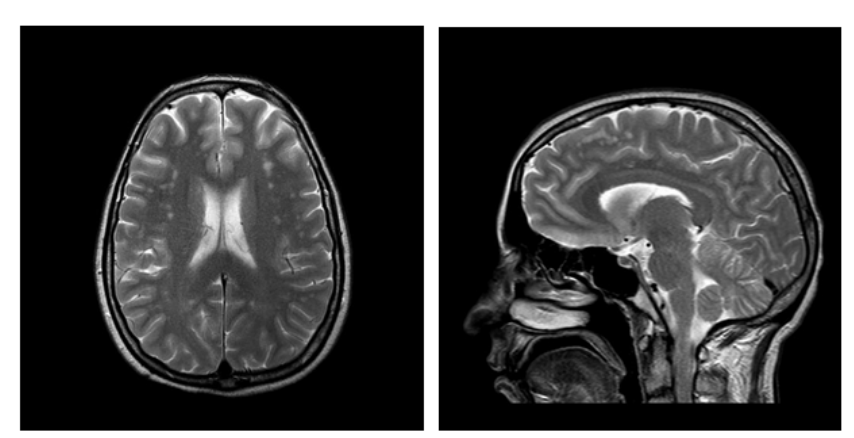

Figure 5 Cranial MRI revealed milimetric hyper-intense lesions on T2-weighted and FLAIR images in the centrum semiovale and periventricular white matter.

Since the patient came from a province where brucellosis was endemic and her Brucella standard serum tube 
agglutination test and Coombs tube agglutination test were both positive (1/80), the T2-weighted hyper-intense lesions on her cranial MRI were interpreted as vasculitic changes of neurobrucellosis.

The patient received 4-month course of combined antibiotic therapy for brucellosis. Her cranial symptoms and complaints dramatically improved. Since the vertebral lesion and the patient's weakness in her legs persisted despite of the brucellosis therapy, the abscess was operated and the frozen section of the lesion was reported as hydatid cyst.

\section{Discussion}

Hydatid cyst is a worldwide zoonosis caused by Echinococcus tapeworms, commonly seen in the Mediterranean region. While liver is the most frequently involved organ, distant anatomic sites such as lung, kidney, spleen, bone and brain might also be involved through hematogenous spread $[2,3]$. Spinal cyst hydatid is the most common bone involvement and it is usually difficult to distinguish from other infectious diseases such as tuberculosis, brucellosis and chronic osteomyelitis in cases of highly destructive processes. Some imaging characteristics of hydatid cyst are absence of intervertebral disc space involvement and vertebral body damage, paraspinal extension and contagious rib involvement $[4,5]$. Although very rare, spinal cyst hydatid might be accompanied by a secondary infection and present like an abscess, making the differential diagnosis almost impossible [6]. In such cases histopathological evaluation is extremely important for the appropriate medical and surgical management.

Brucellosis is another disease commonly encountered in Turkey and the other Mediterranean countries. In \% 5-10 cases, brucellosis might affect the central or peripheral nervous system and present with neurologic clinical manifestations. In addition, imaging abnormalities seen in neurobrucellosis might mimic several neurologic diseases [7]. Inflammation, vascular insult and white matter changes are the imaging abnormalities encountered in neuroburcellosis. There are several patterns of white matter changes that presented with hyper-intense lesions on T2-weighted MR images. Diffuse arcuate fiber region involvement, periventricular pattern, focal demyelinating appearance and nonspecific white matter hyper-intensities are most commonly encountered patterns. After appropriate therapy, complete clinical and radiologic improvement is possible. However, in some patients despite clinical improvement and normalization of CSF after treatment, white matter lesions might not regress or disappear [8-10].

Brucellosis and cyst hydatid might be encountered in the same patient, especially in endemic areas. In our case, neurobrucellosis and spinal hydatid cyst presented at the same time, making the differential diagnosis and prompt appropriate medical and surgical management extremely difficult.

\section{Conclusion}

Hydatid cyst and brucellosis may present in the same patient, at the same time but in different ways. In diagnosis process, if we try to explain all symptoms and lesions based on hydatid cyst or brucellosis solely, we may end up missing one of them.

\section{References}

1. Timur OM, Tanır G, Afsarlar CE, Bayhan GI, Ozgüner IF (2013) Coexistence of liver hydatid cyst and brucellosis in an adolescent. Turkish Parasitology 37: 147-150.

2. Beggs I (1985) The radiology of hydatid disease. AJR Am J Roentgenol 145: 639-648.

3. Lewall DB (1998) Hydatid disease: biology, pathology, imaging and classification. Clin Radiol 52: 863-874.

4. Torricelli P, Martinelli C, Biagini R (1990) Radiographic and computed tomographic findings in hydatid disease of bone. Skeletal Radiol 19: 435-439.

5. Ogut AG, Kanberoglu K, Altug A, Cokyüksel O (1992) CT and MRI in hydatid disease of cervical vertebrae. Neuroradiology 34: 430-432.

6. Kotil K, Tatar Z, Bilge T (2007) Spinal hydatidosis accompanied by a secondary infection. Case report. J Neurosurg Spine 6: 585-590.

7. Al Kawi MZ (1995) Brucellosis. In: Moher JP, Gautier J, (eds). Guide to Clinical Neurology, Churchill Livingstone; New York 677-680.

8. Bashir R, Al Kawi MZ, Harder EJ, Jinkins J (1985) Nervous system brucellosis: diagnosis and treatment. Neurology 35: 1576-1581.

9. Hernandez MA, Anciones B, Frank A, Barreiro P (1988) Neurobrucellosis and cerebral vasculitis. Neurologia 3: 241-243.

10. Al Sous MW, Bohlega S, Al Kawi MZ (2004) Neurobrucellosis: clinical and neuroimaging correlation. AJNR Am J Neuroradiol 25: 395-401. 